\title{
Telerobotics-Assisted Platform for Enhancing Interaction with Physical Environments for People Living with Cerebral Palsy
}

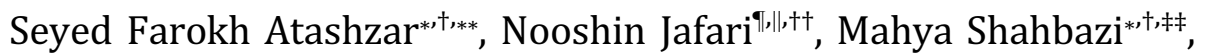

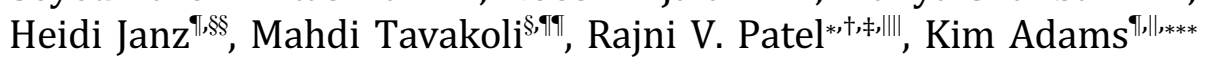 \\ *Canadian Surgical Technologies and Advanced Robotics (CSTAR) \\ London, Ontario, Canada \\ ${ }^{\dagger}$ Department of Electrical and Computer Engineering \\ Western University, London, Ontario, Canada \\ ${ }^{\ddagger}$ Department of Clinical Neurological Sciences \\ Western University, London, Ontario, Canada \\ $\S_{\text {Department of Electrical and Computer Engineering }}$ \\ University of Alberta, Edmonton, Canada \\ TFaculty of Rehabilitation Medicine, Edmonton, Alberta, Canada \\ "Glenrose Rehabilitation Hospital, Edmonton, Alberta, Canada
}

\begin{abstract}
In this paper, the design and implementation of a new telerobotics-assisted platform is proposed for individuals who have cerebral palsy (CP). The main objective of the proposed assistive system is to modulate capabilities of individuals through the proposed telerobotic medium and to enhance their control over interaction with objects in a real physical environment. The proposed platform is motivated by evidence showing that lack of interaction with real environments can develop further secondary sensorimotor and cognitive issues for people who grow up with CP. The proposed telerobotic system assists individuals by (a) mapping their limited but convenient motion range to a larger workspace needed for task performance in the real environment, (b) transferring only the voluntary components of the hand motion to the task-side robot to perform tasks and (c) kinaesthetically dissipating the energy of their involuntary motions using a viscous force field implemented in high frequency domain. Consequently, using the proposed system, an individual who has CP will be capable of providing smooth and large-scale motions and presenting enhanced coordination while performing tasks, even if they naturally have involuntary movements, limited range of motion and/or coordination deficits. The proposed architecture is implemented and initially tested for one nondisabled participant. Afterwards, the system is evaluated for one individual who lives with CP. The resulting quality of motion and task performance are analyzed through a designed clinical protocol. The results confirm the functionality of the proposed assistive platform in enhancing the capabilities of individuals who live with $\mathrm{CP}$ in interacting with physical environments.
\end{abstract}

Keywords: Cerebral palsy; telerobotics; assistive technologies; haptic interaction; involuntary motion extraction.

Received 20 December 2015; Revised 12 March 2016; Accepted 5 April 2016; Published 17 March 2017. Published in JMRR Special Issue on Rehabilitation Robotics. Guest Editors: Loredana Zollo and Jung Kim.

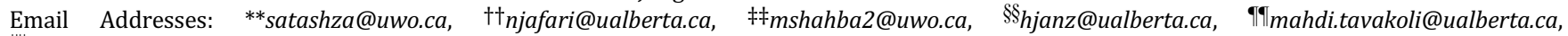
IIIIrvpatel@uwo.ca, ${ }^{* * *}$ kdadams@ualberta.ca

This is an Open Access article published by World Scientific Publishing Company. It is distributed under the terms of the Creative Commons Attribution 4.0 (CC-BY) License. Further distribution of this work is permitted, provided the original work is properly cited.

NOTICE: Prior to using any material contained in this paper, the users are advised to consult with the individual paper author(s) regarding the material contained in this paper, including but not limited to, their specific design(s) and recommendation(s). 


\section{Introduction and Preliminaries}

Cerebral palsy (CP) is an umbrella terminology for a range of nonprogressive neurological sensorimotor deficits that initiate in young children. The onset of $\mathrm{CP}$ is known to be brain damage prior, during and/or after birth. It affects a wide range of motor performances and results in various movement symptoms. Spastic CP, Ataxic CP, and Athetoid CP are some major categories of this condition [1-3]. Note that, in this paper, the terminology "individual with $C P$ " is used for an individual who is living with $C P$. Spastic $\mathrm{CP}$ is a common condition for individuals with $\mathrm{CP}$, which refers to hypertonic muscles that increase muscular tone and result in a reduced range of motion and stiff, jerky or uncoordinated movements [4]. Individuals who have Ataxic CP and Athetoid $\mathrm{CP}$ have reduced or fluctuating muscle tone (hypotonic muscles). Ataxic $\mathrm{CP}$ affects the balance and fine tuning of movements and can involve an intention tremor when attempting movements. Athetoid CP exhibits writhing involuntary movements. Individuals can have two or more types of $\mathrm{CP}$, which is called mixed CP $[1,5,6]$. In summary, different types of $\mathrm{CP}$ involve various motor control impairments including (a) a limited range of motion, (b) coordination problems, and (c) nonsmooth motion execution (such as hand tremor and other involuntary motor behaviors). This condition considerably disturbs the capabilities of individuals with CP in interacting and engaging with their surrounding physical environments [7].

Based on developmental theories, interactional motor experiences, such as exploration and manipulation, are key factors in cognitive and perceptual development of children [8-10]. Children with CP have considerable difficulties in performing object manipulation [11] and may therefore miss the chance for meaningful interaction with environments in early stages of their development $[12,13]$. Consequently, due to the young onset of $\mathrm{CP}$, the limited physical interaction capabilities can result in further secondary conditions such as cognitive development delay, learning deficits, and social skill issues. As an example, movement disorders caused by $\mathrm{CP}$ degrade engagement of young individuals with $\mathrm{CP}$ in free play environments, where children physically interact with objects, make decisions, perform different self-regulated goal-oriented tasks, think about cause-and-effect relationships and understand consequences. In the literature, it has been shown that these actions are crucial for cognitive, sensorimotor, and psychological development of children (see $[12,14,15]$ and the references therein).

Technologies that are capable of allowing disabled people to perform (even indirectly) in a real play environment have attracted interest in recent years. In this regards, play-oriented robots (such as Lego robots) have been studied in the literature. These robots can be controlled using various access methods (e.g. push-down switches or eye gaze) by a child who has disabilities. Corresponding works can be found in a new literature review [12], in [16, 17] and prior research of K. Adams $[14,15,18-20]$. Children with severe physical limitations control the aforementioned robots by high-level supervisory commands. With high-level commands, when a user makes a simple movement (e.g. pushes a switch), the robot performs an autonomous subtask (and usually keeps doing that) while waiting for a new command. Some examples of high-level supervisory commands are reach the target, drop the object, and come back to home position. An example of a switch controlled robot system is shown in Fig. 1.

Although the users are able to perform some tasks using the above-mentioned systems, there is still a considerable lack of controlled, direct, instant and coordinated interaction between the user and the play environments [21]. In other words, although these systems can indirectly realize task performance in play environments, they are incapable of realizing motioncontrolled hand-eye coordinated physical interaction with real objects and they do not correlate the user movement (e.g. pushing a switch) with interaction in the environment (the robot performs a multi-step task).

Remark 1.1. Using the multi-action guidance delivered by the proposed system, the motions of the task-side robot can be controlled by the disabled user and would be correlated to the motion of the user's hand. For example, if the user tries to move the user-side interface (e.g. a motorized haptic device) to the left, the task-side robot correspondingly and instantly will go to the left. If children could exert controlled coordinated physical interaction to manipulate objects in the environment, it is anticipated that it could lead to positive effects on their cognitive, social and sensorimotor development in the long term.

Remark 1.2. In this paper, we propose a new hapticsenabled telerobotic platform that can help individuals with $\mathrm{CP}$ in interacting with real physical environments mostly by augmenting their motion capabilities and also by making it possible for them to feel the interaction forces. When interacting with play objects, individuals need to be able to reach them, use smooth and fluid movements, and perform hand-eye coordination with the appropriate force and/or speed [22]. The goal of the proposed system is to allow individuals with $\mathrm{CP}$ to perform instant under-control tasks through a telerobotic medium that can compensate for the limited range of motion, the nonsmooth tremor (and/or involuntary actions), and coordination problems that individuals with-CP may exhibit. This system makes it possible for individuals with $\mathrm{CP}$ to utilize their own movement strategies and motion capabilities and instantly interact with various physical environments. The main responsibility of the proposed telerobotic system is to extend the 


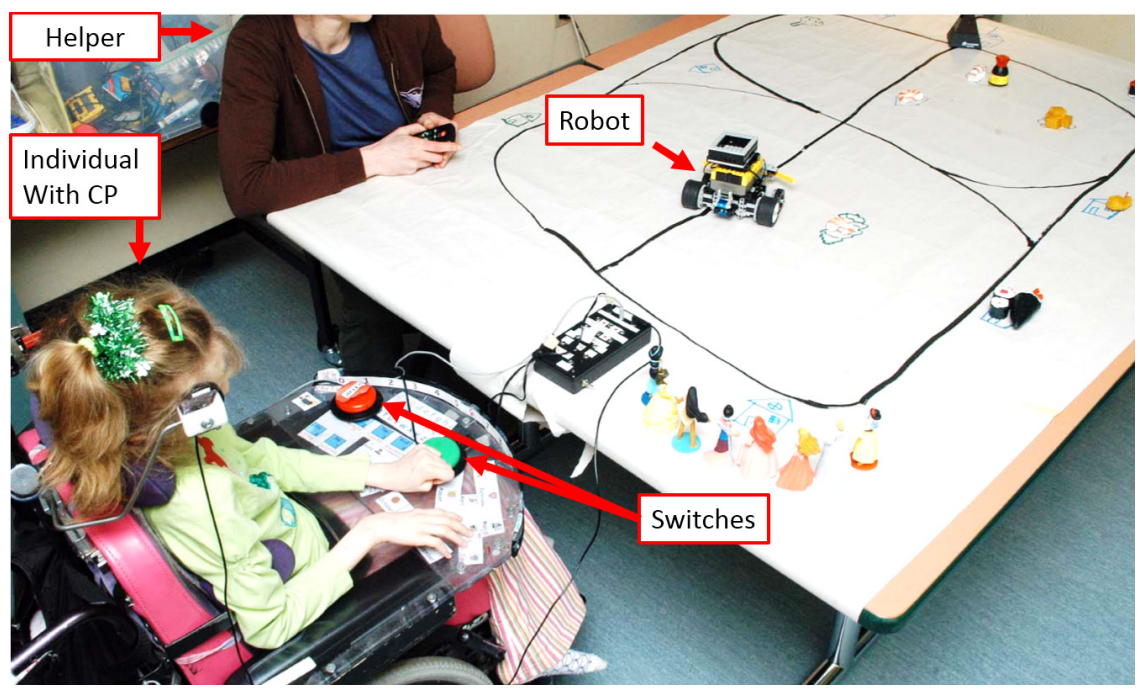

Fig. 1. An example of current systems used for individuals with CP.

capabilities of individuals with $\mathrm{CP}$ and allow them to experience interaction with environments.

Remark 1.3. The main focus of this paper is to propose a new mechatronic design which can facilitate interaction with physical environments for individuals with $\mathrm{CP}$, as a new assistive paradigm. The design is motivated by evidence showing that physical interaction is a crucial factor for developing sensorimotor and cognitive skills, and that individuals living with a lack of interaction may develop corresponding secondary delays [8-10, 12, 13]. Analyzing the long-term effectiveness of the proposed system to cognitive and sensory development will require longitudinal long-term studies, which are part of our ongoing work, but outside of the scope of this paper. $\bullet$

It should be noted that telerobotic systems have been utilized in a wide variety of applications such as surgical, under-water and space operations [23]. A common goal for telerobotic systems is to extend the capabilities of human users beyond their limitations. In addition, it is possible to quantify movement capabilities (such as for skill assessment during robotics-assisted surgery) [23]. Using the same concept, in this paper, the new telerobotic system is proposed to help disabled users to extend their capabilities beyond limits imposed by their movement disorders to allow them to experience interaction with environments while the system logs all motion and force profiles.

\subsection{Overview of the proposed system}

The telerobotic architecture proposed in this paper consists of (I) a user-side robot, (II) a task-side robot, (III) a Virtual Assistive (VA) computer algorithm, and (IV) a physical play environment. The system architecture has two major signal pathways namely: forward path and backward path. In the forward path, (a) the individual with CP generates motions by moving the user-side robot, (b) the VA algorithm modifies the generated motion (to enhance the task performance), and (c) the task-side robot mimics the motions modified by the VA algorithm to perform the task on the play environment. In the backward path, the VA algorithm generates a resistive compensatory force field (explained later), which is applied by the user-side robot to the individual's hand to kinaesthetically restrict his/her involuntary movements. Consequently, the proposed system is a medium which makes it possible for individuals with $\mathrm{CP}$ to interact with a play environment (placed at the location of the task-side robot), while the system compensates for their limited range of motion and nonsmooth movements. In addition, it provides a compensatory force field for the user to kinaesthetically restrict the involuntary movements. The mentioned functionality allows an individual with CP to engage in interactions with environments that they cannot engage in without the use of this system. A schematic of the proposed telerobotic system is shown in Fig. 2.

The proposed architecture has a triple-action design as follows:

Action \#1: Motion Range Correction: Scale the user's limited convenient range of motion at the user-side robot to that needed for performing the task at the task-side robot.

Action \#2: Voluntary Movement Tracking: Filter out the motion signal transferred to the task-side robot in order to only use the voluntary component of the provided motion for task performance.

Action \#3: Involuntary Movement Dissipation: Apply a dissipative force field to the user's hand by the user-side robot to damp-out and resist the high-frequency involuntary component of the hand motion while keeping the 


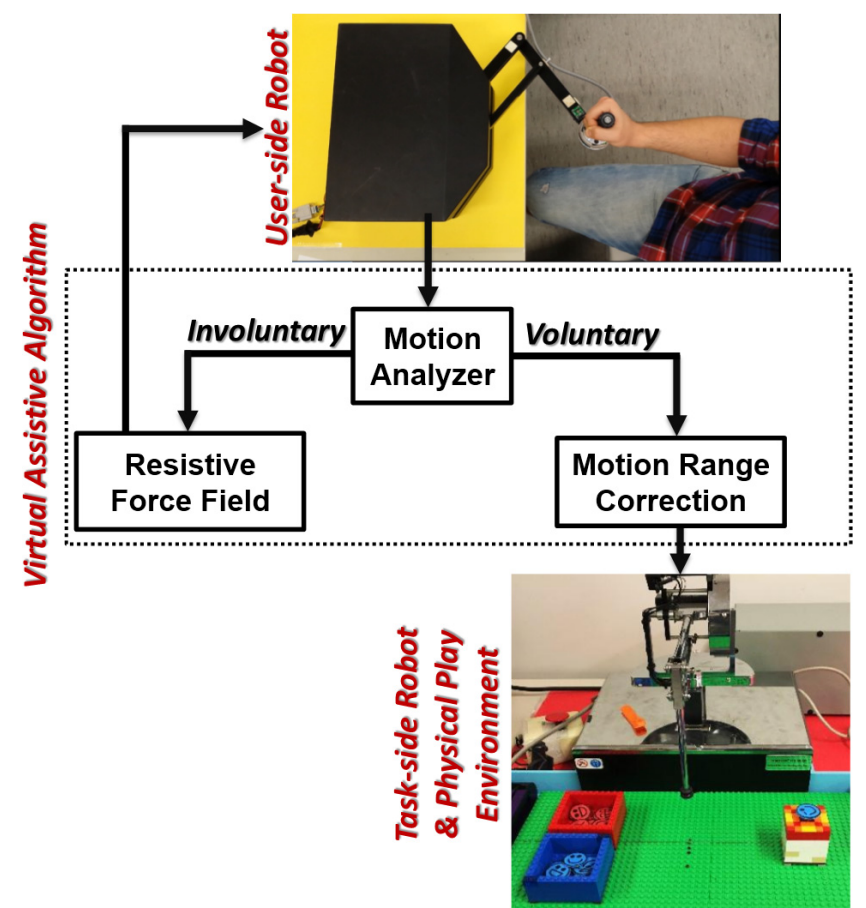

Fig. 2. The experimental setup and a schematic of the proposed triple-action telerobotic architecture. The user side robot is a table-top rehabilitation robot from Quanser Inc. (Canada) and the task-sided robot is a Phantom Premium $1.5 \mathrm{~A}$ from Geomagic (US).

dissipative resistance small for the voluntary component (that is typically a low-frequency signal $[24,25]$ ).

The play environment can support many tasks like sorting objects, path following, obstacle avoidance or push-pull tasks. In order to verify the functionality of the system, the proposed architecture was implemented using a table-top upper-limb rehabilitation robot (from Quanser Inc., Canada) as the user-side device, and a Phantom Premium 1.5 A robot (from Geomagic, US) as the task-side device. Each action of the implemented system was evaluated with one nondisabled subject. The implemented system was demonstrated to therapists and their feedback was utilized to optimize the design and make the protocol of this study. Using the designed protocol, the system was tested with one individual with CP. Force and motion trajectories were collected to analyze the performance of the system.

The rest of this paper is as follows. In Sec. 2, the design of the telerobotic system and the proposed virtual assistive algorithm are explained in detail. In addition, the triple-action performance of the proposed architecture is evaluated for the nondisabled individual. In Sec. 3, the clinical evaluation method is introduced to evaluate the effectiveness of the system for the individual with CP. The results and discussion are given in Sec. 3. The paper is concluded in Sec. 4.

\section{The Proposed Telerobotic Architecture and Virtual Assistive Algorithm}

The design of the proposed telerobotic system shown in Fig. 2 is described in more detail, in this section, and experimental results are reported to evaluate different functions of the system.

\subsection{Components and the design of actions}

The components of the designed system are as follows.

(a) User-side Robot: The user-side robot is a 2 degrees of freedom upper-limb rehabilitation robot (from Quanser Inc., Markham, Ontario, Canada). The specification of the robot (including the shape and size of the workspace, resolution, weight, etc.) can be found in $[26,27]$. The robot can apply forces up to $50 \mathrm{~N}$ in Cartesian domain. The function of the user-side robot is to register the individual's hand motion and provide him/ her with a compensatory force field generated using the Virtual Assistive algorithm, detailed later in this section.

(b) Task-side Robot: The task-side robot is a Phantom Premium 1.5A robot (from Geomagic, US). The specifications can be found in [28]. The task-side robot is considered to follow the "modulated" motion trajectories of the individual with $\mathrm{CP}$ in order to perform the intended task. In this paper, one of the considered functional tasks is a pick-and-place sorting activity explained latter in this section. A small electromagnetic lifter is attached at the tip of the task-side robot to perform the task. The motion trajectory to be followed by the task-side robot is based on the individual's hand motion and is modulated by the VA algorithm.

(c) Virtual Assistive Algorithm: The VA is an algorithm that controls the behavior of both the user-side and the task-side robots. The algorithm first analyzes the individual's hand motion and extracts the involuntary and voluntary components. For this purpose, a low-pass Band-limited Multiple Fourier Linear Combiner (BMFLC) filter (see [29-32]) is implemented to extract the involuntary component of the hand motion, which has a highfrequency nature in comparing with voluntary component of the motion $[24,25]$. The BMFLC filter is an adaptive technique which is utilized to extract involuntary movements while introducing minimal filtering latency into the interaction. The design of the filter can be found in [29-32].

Remark 2.1. The BMFLC filter used is a member of a family of adaptive filters which are designed based on Fourier Linear Combiner (FLC) modeling. This family considers summation of discrete harmonics over a large window of frequencies (for example from $0 \mathrm{~Hz}$ to $20 \mathrm{~Hz}$ ) for modeling a signal that has high-frequency and low-frequency components. The filter adaptively and in 
real-time tunes the coefficients of the FLC model, using a recursive technique which is usually the Least-MeanSquare (LMS) technique, to find the best frequency-based decomposition of the targeted signal. FLC-based filters have demonstrated good performance in extracting involuntary movements introducing minimum delay in comparison with classical filtering techniques [29-31, $33,34]$. The original format of FLC-based filters assumes a single dominant frequency [35] for the signal to be filtered. This was utilized to extract and cancel out physiological hand tremor of surgeons in the development of motorized surgical tools with the goal of increasing accuracy in surgical tasks [34]. The abovementioned assumption (single dominant frequency) was then relaxed by the newer version of the filter i.e. the BMFLC technique. BMFLC is designed to track multiple harmonics of a signal [29-31] and showed higher accuracy compared to conventional FLC-based filters [30]. In the literature, BMFLC filtering has also been used for extracting physiological hand tremor of surgeons [36-38] and recently has shown good performance in extracting pathological involuntary motions [33].

Using the BMFLC filter, the extracted involuntary and voluntary motions are

$$
\begin{gathered}
\xi_{i}(t)=\operatorname{BMFLC}\left(M_{p}(t)\right), \\
\xi_{v}(t)=M_{p}(t)-\xi_{i}(t),
\end{gathered}
$$

respectively. In (1) and (2), $M_{p}(t)$ is the total hand motion which has both of the voluntary and involuntary components. In addition, $\xi_{v}(t)$ is the estimated lowfrequency voluntary component of the hand motion and $\xi_{i}(t)$ is the estimated involuntary component. When $M_{p}(t)$ is considered to be the hand "velocity", then $\xi_{v}(t)$ and $\xi_{i}(t)$ are voluntary and involuntary component of the hand's velocity, respectively. The same interpretation is valid for position trajectories.

After estimating $\xi_{v}(t)$ and $\xi_{i}(t)$, the VA provides the three actions, explained in the following.

Action \#1: Motion Range Correction: The algorithm provides range correction to the transferred motion. For this purpose, prior to the task execution, the system operator asks the individual with CP to explore the limits of his/her comfortable range of motion using the userside robot. The maximum range of motion needed to perform the task is known based on the specific dimensions of the task environment. Accordingly, the needed scaling can be calculated for the individual with $\mathrm{CP}$, on a user-specific basis, to map his/her comfortable motion range to the one needed for task performance. For this purpose, scaling factors $C_{x}$ and $C_{y}$ are calculated as follows (for 2D tasks):

$$
C_{x}=\frac{\operatorname{Max}\left(X_{p}\right)}{\operatorname{Max}\left(X_{T}\right)},
$$

$$
C_{y}=\frac{\operatorname{Max}\left(Y_{p}\right)}{\operatorname{Max}\left(Y_{T}\right)} .
$$

In (3), $C_{x}$ is the mapping coefficient for the $X$-direction that relates the maximum reachable distance of the individual's hand $\left(\operatorname{Max}\left(X_{p}\right)\right)$ in his/her comfortable range of motion to the maximum amplitude of motion needed in the $X$-direction to perform the designed task $\left(\operatorname{Max}\left(X_{T}\right)\right)$. Note that $X_{p}$ stands for the individual's hand position in the $X$-direction and $X_{T}$ stands for the taskside robot movement in the $X$-direction. Accordingly, $C_{y}$ is designed similarly for the motion in the $Y$-direction. Consequently, the range-corrected motion trajectory for the task-side robot is

$$
M_{s}(t)=\left(\begin{array}{cc}
C_{x} & 0 \\
0 & C_{y}
\end{array}\right) \cdot \xi_{v}(t)^{T} .
$$

In (5), $\xi_{v}(t)$ refers to the voluntary component of the individual's position trajectory in 2 degrees of freedom $(X-$ and $Y$-directions). The task-side robot follows the corrected (scaled-up) trajectory of the individual's hand. Consequently, the individual with $\mathrm{CP}$ will be able to perform large-scale tasks using her/his comfortable workspace range. In Sec. 3, a systematic approach is proposed to tune default values for $C_{x}$ and $C_{y}$. In addition, explanations are provided to show how to choose the cut-off frequency of the BMFLC filter to separate the voluntary and involuntary movements. Note that, the clinician will be always able to tune these parameters. This is a widely utilized approach in the literature $[39,40]$.

Action \#2: Voluntary Movement Tracking: In order to provide better coordination for the individual with $\mathrm{CP}$, instead of transferring the total movement of the hand (i.e. $M_{p}(t)$ ), only the voluntary component $\xi_{v}(t)$ is considered to be sent to the task-side robot. The goal is to make the robot follow the voluntary component of the hand motion to perform the task. Consequently, when an individual who has CP moves the user-side robot with a movement containing both low-frequency movements (i.e. a movement generally towards the target) and high frequency movements (i.e. a jerky or shaky movement), only the low frequency movements will be transferred to the task-side robot.

Action \#3: Involuntary Movement Dissipation: The goal of the third action is to provide the individual with CP with resistive dissipative forces in the high frequency range to damp-out the energy of his/her involuntary hand motion. This provides the user with better coordination of the task and smoother, controlled motions. In addition, using this action, a better hand-eye coordination will be provided for the individual with $\mathrm{CP}$. The reason is that using this action, the involuntary component of the hand motion dampens out, which matches the visual feedback from the task-side robot controlled to 
track the voluntary component of hand motion (based on Action \#2). In order to implement Action \#3, the involuntary component of the hand motion, which is extracted by the BMFLC filter, is utilized in the design of the resistive dissipative force field. It should be highlighted that the force field is implemented in the high-frequency domain and provides the user the feeling of moving in a viscous environment only for the involuntary component of the user's motion. Consequently, the force field provides minimal to no resistance in response to the voluntary components of the user's movements. The design of the proposed force field is as follows:

$$
F_{i}(t)=\left(\begin{array}{cc}
B_{x} & 0 \\
0 & B_{y}
\end{array}\right) \cdot \xi_{i}(t)^{T} .
$$

In (6), $F_{i}(t)$ is the designed force field, $B_{x}$ and $B_{y}$ are dissipation coefficients that define the intensity of the resistive force field. The higher the intensity, the higher the magnitude of the generated resistive forces in response to the same input (i.e. involuntary component of hand velocity). It should be noted that since the dissipation coefficients are applied to the involuntary velocity of the individual's hand at the user-side robot, the intensity of the dissipation converges to zero for voluntary movements.

Remark 2.2. Note that $B_{x}$ and $B_{y}$ define the intensity of the resistive force field. Feedback from the patient and clinicians is considered to tune these parameters. The reason is that the intensity delivered using the proposed architecture dependents on (a) the musculoskeletal power of the patient, (b) the level of intensity that the therapist/clinician would like to deliver to the individual's hand, and (c) the level of comfort that the individual feels during the trial. Considering feedback from a therapist/clinician is common for neuro-rehabilitation robotic systems, where the therapist/clinician gradually tunes the difficulty level while the patient performs tasks [39, 40]. As a result, we suggest to allow the clinician to tune $B_{x}$ and $B_{y}$ based on their understanding of the needs of the individual with $\mathrm{CP}$ and considering the individual's comfort level during the interaction. The tuning procedure is explained in more detail in Sec. 3. •

(d) Task Environment: The other component of the proposed telerobotic architecture is the designed functional task environment. The geometry of the environment is decided considering the motion-capabilities of the task-side robot. Also, specific attention is paid to make the task environment "flexible". Our clinical partners expressed that the environment needed to be flexible in order to quickly change the tasks based on the needs and interests of the individual with $\mathrm{CP}$ and the therapist. In this paper, one of the main implemented tasks was to sort 10 magnetic happy face objects based on their color when the initial pick-up position and the target drop-off locations were separated by $33 \mathrm{~cm}$ in the $X$-direction. There were two side-by-side target bins one for the red happy faces and one for the blue ones. The individual needed to use the proposed telerobotic system to sort the magnetic objects. This task is shown in Fig. 2 in the physical play environment. This is one example possible tasks and there are more possibilities. Sorting can also help children to learn about the attributes of objects (like color and shape), as they sort them into categories.

Remark 2.3. Although the functionality of different actions of the system can be evaluated separately, the actions are designed to work simultaneously for individuals with CP. The reason is that if we just utilize Action \#1 (scaling up the motions), we amplify not only the individual's voluntary motions, but also the involuntary motions. In this case, the individual with CP would have more difficulty controlling the task-side robot. That is why the proposed architecture is designed to only assist the voluntary component while kinesthetically resisting and avoiding tracking the involuntary components of motion. •

\subsection{Experimental evaluation}

In this part, data from the experimental evaluation with a non-disabled user of the three actions of the proposed VA algorithm are presented. The experiments were performed in three phases where each phase corresponds to one of the defined actions.

Phase 1: Experiment for Motion Range Correction (Action \#1): This phase was designed to evaluate the functionality of the proposed Action\#1 of the developed VA. The experiment started at $t=20 \mathrm{~s}$. First, the operator moved her hand for $30 \mathrm{~s}$ while the scaling factors for both $X$ - and $Y$-directions were equal to unity. The generated motion trajectory of the user is compared to the one followed by the task-side robot in $X$ - and $Y$-directions in Figs. 3(a) and 3(b), respectively. Also, the 3D trajectory is shown in Fig. 3(c). As expected, the taskside robot tracked the trajectories generated by the user. For the second part, at $t=70 \mathrm{~s}$, the corrective gains $C_{x}$ and $C_{y}$ were increased by $50 \%$. The trajectories for the $t \geq 70 \mathrm{~s}$ can also be seen in Fig. 3. As expected in this condition, the task-side robot followed the scaled-up trajectory even though the user performed smaller motions.

Phase 2: Experiment for Voluntary Movement Tracking (Action \#2): In this phase, to only evaluate the performance of Action \#2, the dissipative force field was disabled. The force field is exclusively studied in the third phase of this experiment. First, the nondisabled operator provided motions only in a high-frequency manner in the $X$-direction. 


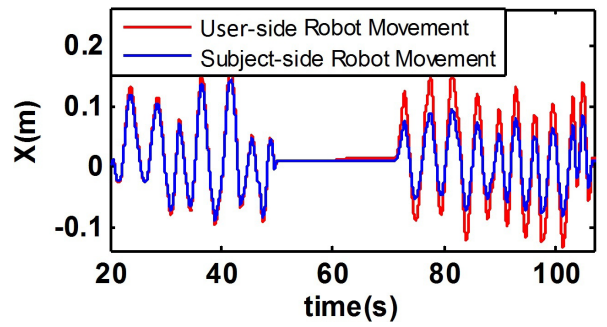

(a)

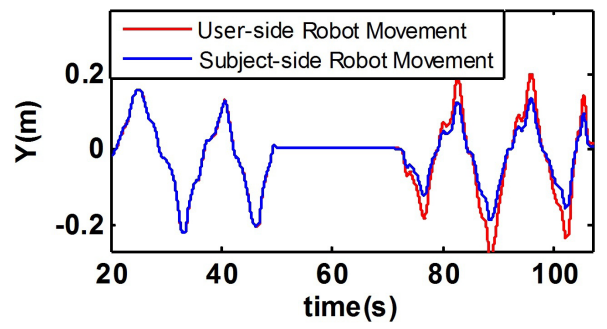

(b)

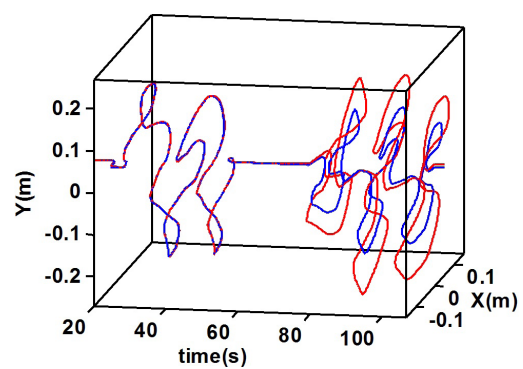

(c)

Fig. 3. Motion trajectories for evaluating Action \#1 (i.e. Motion Range Correction): (a) X-direction, (b) $Y$-direction and (c) 3D view.

Since the high-frequency behavior was provided by a human operator (not programmed software), it is not possible to report the frequency. However, providing motions this way enabled testing the performance of the proposed action. It was expected that the task-side robot would not move much due to the proposed Voluntary Movement Tracking action of the system (Action \#2). Next, the operator provided motions in a low-frequency manner. It was expected that the task-side robot would track the user-side robot, since applying Action \#2, the low-frequency movements should pass through the VA algorithm. Then, the operator provided a hand motion which had both high-frequency and low-frequency components. It was expected that in this case, the robot would follow only the low-frequency component.

The results are shown in Fig. 4, which confirms the functionality of Action \#2 of the proposed VA algorithm. Considering Fig. 4(a), when the motion had mostly high-frequency components, the task-side robot did not move much. However, when the operator moved the robot in a low-frequency manner, the task-side robot followed the generated trajectory in Fig. 4(b). When the motion had both high and low frequencies, the task-side robot mainly followed the low-frequency component, as shown in Fig. 4(c). Since perfect filtering is not theoretically possible, some small high-frequency components can still be observed in Figs. 4(a) and 4(c).

Phase 3: Experiment for Involuntary Movement Dissipation (Action \#3): In this phase of the experiment, the dissipative force field (designed for suppressing involuntary motion through delivering a viscous force field in high-frequencies, applied by the user-side robot) was enabled and the same procedure as the one given in Phase 2 was repeated. Consequently, during the first part of the experiment, the nondisabled operator moved the robot in a high-frequency manner. Afterwards,

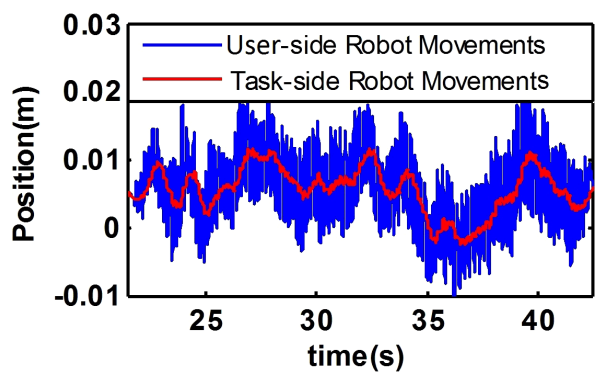

(a)

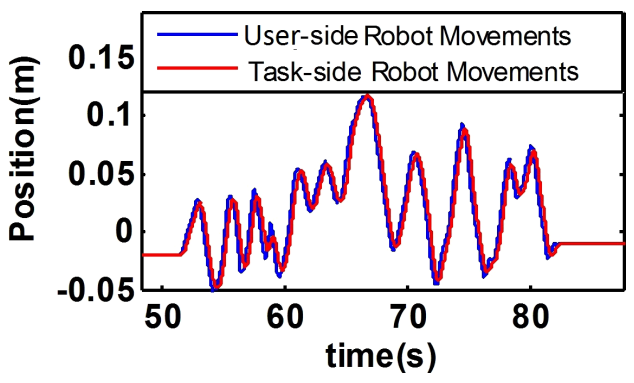

(b)

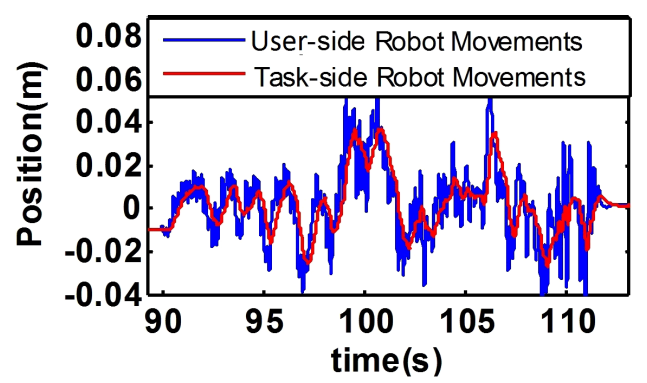

(c)

Fig. 4. Motion trajectories in the $X$-direction: The user provided (a) high-frequency movements (first part), (b) low-frequency movements (second part) and (c) movements that had both high-frequency and low-frequency components (third part). 


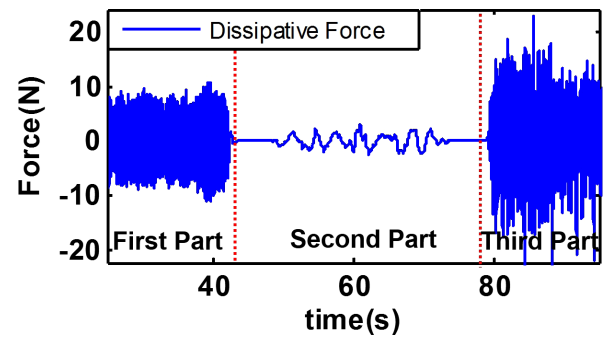

(a)

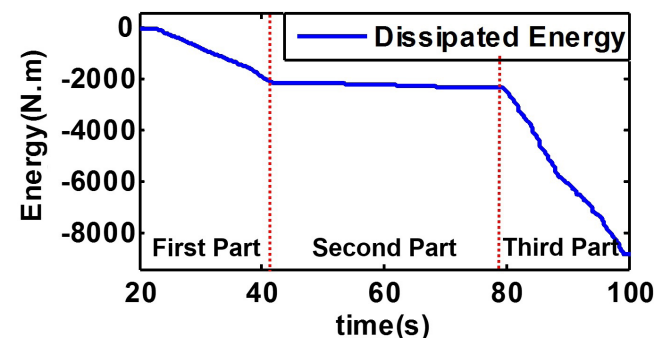

(b)

Fig. 5. (a) The generated force to damp-out the energy of the involuntary movements and (b) the corresponding energy curve.

during the second part of the experiment, she moved the robot in a low-frequency manner, and finally during the third part of the experiment, she moved the robot in a mixed-frequency manner (which included both high-frequency and low-frequency components). It was expected that the user would feel high amplitude compensatory resistive forces in the first part (when only high-frequency motions are applied) and the third part (when mixed-frequency motions are applied) of this procedure, thus dampening the energy of the high frequency component. It was also expected that the user would not feel much resistance during the second part of this experiment. The generated force (for all the three parts) is shown in Fig. 5(a). As can be seen in the figure, the designed VA algorithm has applied high amplitude forces in the first $(t<45 \mathrm{~s})$ and third $(t>80 \mathrm{~s})$ parts when there was high-frequency involuntary components in the motion. The dissipating energy provided by the proposed VA algorithm is given in Fig. 5(b). The energy is calculated as follows:

$$
E_{\mathrm{dis}}(t)=\int_{0}^{t} F_{i}(\tau) \cdot \xi_{i}(\tau)^{T} d \tau
$$

Using (7), a high negative slope means a high rate of energy dissipation and a positive slope means energy generation over time. Considering Fig. 5(b), during the first and the third parts of the experiment, the system considerably dissipates the interaction energy (the average slope for the first part was $-105 \frac{\mathrm{N} \cdot \mathrm{m}}{\mathrm{s}}$ and for the third part was $-336 \frac{\mathrm{N} \cdot \mathrm{m}}{\mathrm{s}}$ ) while during the second part of the experiment the energy dissipation was close to zero (the average slope was $-4.3 \frac{\mathrm{N} \cdot \mathrm{m}}{\mathrm{s}}$ ).

As can be seen in Fig. 5, the designed VA algorithm has met our expectations and has dissipated the energy of the high-frequency component of the motion (in the first and the third parts of this phase) and not the lowfrequency one (the second part). This can be seen by the negative slope of the energy curve during the first and the third parts, while the slope is almost zero during the second part. This experiment confirms the functionality of the proposed Action\#3 of the VA algorithm.

The above-mentioned experiments and results confirm the functionality of the proposed system and shows that the implemented VA algorithm and the proposed telerobotic architecture are performing as expected during the experiments involving a nondisabled user.

\section{Clinical Protocol, Results and Discussion}

In order to evaluate the effectiveness of the proposed architecture in assisting individuals with $\mathrm{CP}$, the following protocol was conducted with one individual with CP. The ultimate goal of the protocol was to analyze the performance of the individual with $\mathrm{CP}$ when the three actions were enabled (modulated interaction) in comparison to the situation when the actions were disabled (normal interaction).

The individual is an adult with mixed CP which affects her upper and lower limbs. She is classified as Level IV in the Gross Motor Function Classification System Expanded and Revised (GMFCS-E\&R) [41], meaning she can perform self-mobility with limitations (she uses a powered wheelchair). On the Manual Ability Classification System (MACS) [42], she is classified at Level III, meaning she can handle objects with difficulty, and needs help to prepare and/or modify activities.

Step 1: Preparation-Part\#1 (Familiarization): During the first step, the individual with $\mathrm{CP}$ was given time to get familiar with how moving the user-side robot resulted in movements of the task-side robot, and how the user-side robot exerted forces. The goal was to minimize the potential effects of adaptation on the results.

Step 2: Preparation-Part\#2: Workspace Identification for Motion Range Correction (needed for Action \#1): During the second part of the preparation, the individual with CP was asked to explore the boundaries of her convenient workspace. This was done to find the needed scaling factors so that the individual could reach the targets in the task. The convenient motion range, and the needed workspace to perform the task are shown in Fig. 6. Based on (3) and (4), the results show that the participant needed 1.65 scaling up for the 


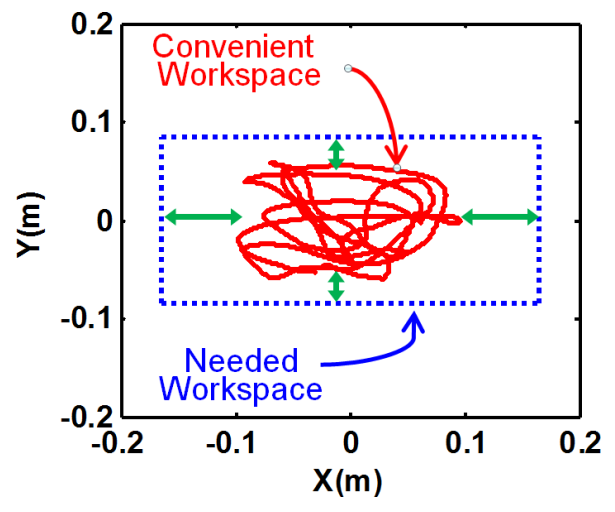

Fig. 6. The convenient workspace versus the needed workspace.

$X$-direction and 1.3 scaling up for the $Y$-direction. In other words, considering the designed workspace, CP has reduced the participant's motion range in the $X$-direction by almost $40 \%$ and in the $Y$-direction by almost $33 \%$.

Step 3: Preparation-Part\#3: Filtering Frequency Identification (needed for Actions \#2 and \#3): In order to find the cut-off frequency for the BMFLC filter, which is utilized to implement Actions \#2 (Voluntary Movement Tracking) and Action \#3 (Involuntary Movement Dissipation), the hand velocity of the individual with CP was analyzed. For this purpose, two objects were placed on the boundaries of the designed workspace and the individual with CP moved the user-side robot so that the task-side robot moved between the objects repetitively for $30 \mathrm{~s}$. The frequency power spectrum is shown in Fig. 7 for the $X$-direction motion. Based on the results, the cut-off frequency (i.e. the frequency that can separate voluntary and involuntary motions) was calculated as $0.7 \mathrm{~Hz}$ which is shown by the black line in Fig. 7. In this paper, the cut-off frequency was manually selected as the value between the main high and low frequency peaks.

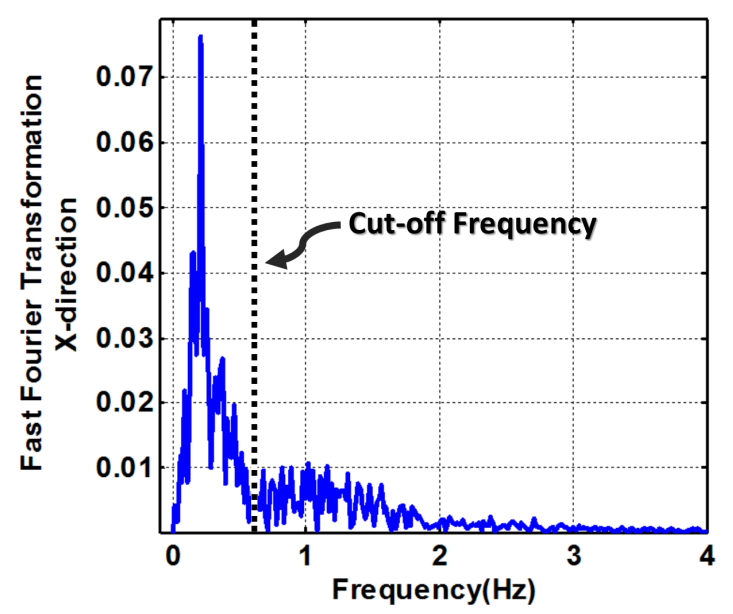

Fig. 7. Analysis characteristics of the motion in the frequency domain.
Step 4: Preparation-Part\#4: Dissipation Gain Identification for Involuntary Movement Energy Dissipation (needed for Action \#3): In this part of the experiment, the dissipation gain in (6) was increased by $20 \mathrm{~N} \cdot \mathrm{s} / \mathrm{m}$ steps from $0 \mathrm{~N} \cdot \mathrm{s} / \mathrm{m}$ to $100 \mathrm{~N} \cdot \mathrm{s} / \mathrm{m}$, while the user performed random movements at the user-side robot. The goal was to determine the best dissipation gain chosen based on observations of the user's performance and the individual's feedback. The individual with CP moved the user-side robot at each dissipation gain, and rated the perceived exertion from: "easy, slightly difficult, fairly difficult, difficult, and very difficult". A dissipative gain of $40 \mathrm{~N} \cdot \mathrm{s} / \mathrm{m}$ for both the $X$ - and $Y$-directions was selected for the study, as it was the value preferred by the individual that could provide enough control over the task for her. Feedback from a therapist could also be helpful to tune this factor. After finding the parameters, in order to evaluate the effectiveness of the system in smoothing the movement trajectories and enhancing coordination accuracy, a twophase clinical task was designed, as explained in Steps 5 and 6.

Step 5: Clinical Task- Phase \#1 (Navigation Game): For this task, the user navigates the task-side robot between three locations. Two targets were located at the boundaries of the workspace (i.e. the ferromagnetic object pick-up location at the right and between the dropoff bins at the left in Fig. 1). The two targets were separated by $33 \mathrm{~cm}$ in the $X$-direction. The third target was a home position between the two targets. The individual with CP moved the task-side robot (through the telerobotic medium and by providing motion at the user-side robot) from the left target to the home position, and then from the home position to the right target, and vice versa. She was asked to try to stay on each target for $4 \mathrm{~s}$. She was given verbal cues about the timing of movement. This experiment was designed to analyze accuracy and smoothness. The individual with CP repeated this motion eight times, first with all the three proposed Actions of the system disabled and then eight times with all of the Actions enabled. The targeting accuracy and motion smoothness are shown in Figs. 8 and 9. As can be seen in Fig. 8, the targeting accuracy, which is measured as the distance from the center of the target averaged over the $4 \mathrm{~s}$, is considerably enhanced when the three actions were enabled (modulated interaction), in comparison to when the actions are disabled (normal interaction).

In addition, the Fast Fourier Transform frequency content analysis of the movement velocity on the userside robot, given in Fig. 9, shows that when the proposed three actions of the system are enabled, the power spectrum of the movement velocity contains lower frequency components whereas considerable more highfrequency components can be observed when the proposed three actions of the system are disabled. This 


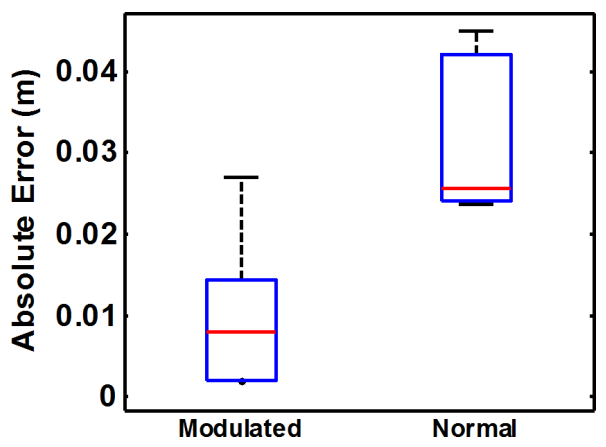

Fig. 8. Absolute error, using the modulated interaction (when the proposed three actions of the system are enabled), and for the normal interaction (when the proposed actions are disabled).

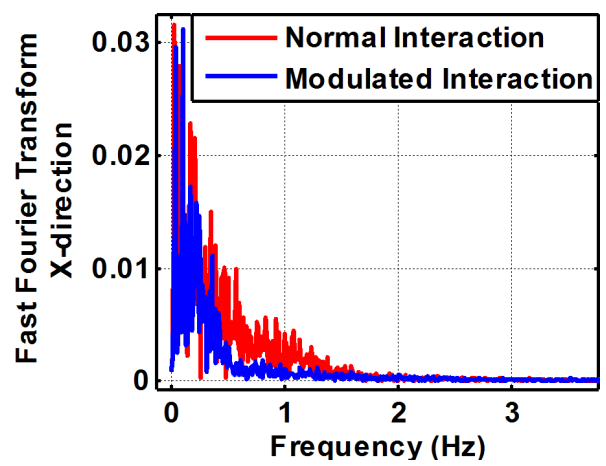

Fig. 9. Navigation Game: Analysis characteristics of the movement in the frequency domain for both modulated interaction (when the proposed three actions of the system are enabled) and for the normal interaction (when the proposed three actions are disabled).

result confirms the motion smoothing feature of the system for task performance. It should be highlighted that the low-frequency components of the movement velocity for the case of modulated interaction is close to the normal interaction. This means that the system does not considerably affect the low-frequency movements while the high-frequency involuntary component of the movement is dissipated. In summary, the movements are smoother and the coordination is more accurate, which serves the very purpose of the proposed architecture.

Step 6: Clinical Task- Phase \#2 (Pick and Place Game): In order to better evaluate the functionality of the system, the second phase of the clinical task was developed. In this phase, the individual with $\mathrm{CP}$ was asked to sort the ferromagnetic happy face objects based on their colors (red or blue). For this purpose, as mentioned before, on the right side of the designed workspace, an initial position is considered for the magnetic happy faces. On the left side of the workspace, two colorcoded target locations for the objects were placed sideby-side. The designed workspace can be seen in Fig. 2 .
The initial position and the targets are separated by $33 \mathrm{~cm}$ in the $X$-direction. In total, the user sorted 10 objects. The individual was asked to placed the objects in the corresponding target position one after the other. A research assistant put the magnetic happy faces on the initial pick-up location (one by one).

The experiment was conducted successfully and the individual with $\mathrm{CP}$ was able to sort the objects while the system smoothed her motions and increased her movement range, which ultimately resulted in a more accurate coordination and control over the task execution, despite the fact that the targets were placed out of her convenient workspace.

The resulting movement trajectories were analyzed in the frequency-domain (as shown in Fig. 10) similar to the previous step of the study (Navigation game). In addition, velocity profiles of the modulated interaction and the normal interaction are compared in Fig. 11. Also, movement in the $X$ - and $Y$-directions together with the 2D movement trajectory are shown in Fig. 12 (for the case of modulated interaction) and in Fig. 13 (for the case of normal interaction).

As it can be seen in Fig. 10, the involuntary components of the motion are considerably dissipated by the use of the proposed modulated interaction in comparison with the normal interaction. Also the use of the proposed modulated interaction has resulted in smoother position and velocity trajectories, which can be seen in Fig. 11 for the movement velocity profile and can be also observed by comparing Figs. 12 and 13 for the movement position profile.

In addition, for the case of modulated interaction, the motion execution (moving from the home position to the target) conducted by the individual with $\mathrm{CP}$ is more coherent. This can be seen by comparing the $2 \mathrm{D}$ motion in Figs. 12(c) and 13(c), and also by comparing the 2D histograms of the motion trajectories shown in Fig. 14. It

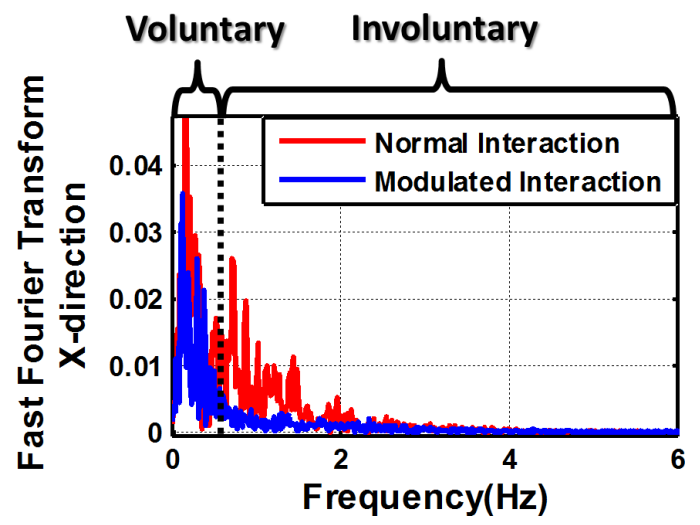

Fig. 10. Pick and Place Game: Analysis of characteristics of the movement in the frequency domain for both modulated interaction (when the proposed three actions of the system are enabled) and for the normal interaction (when the proposed three actions are disabled). 


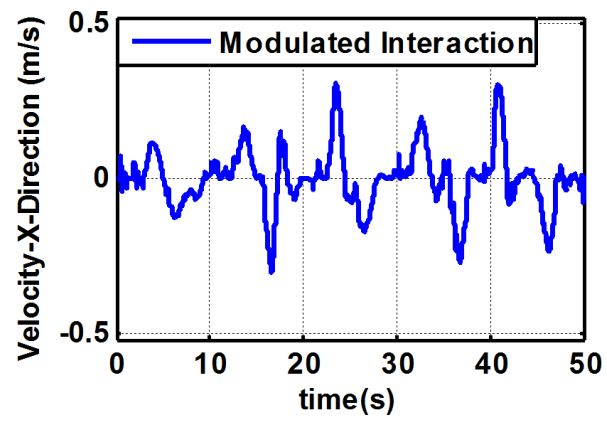

(a)

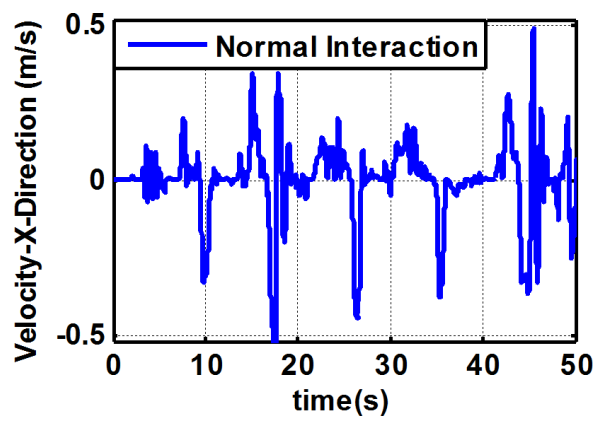

(b)

Fig. 11. Movement velocity in the $X$-direction: (a) for the case of modulated interaction and (b) for the case of normal interaction.

should be noted that as shown in Fig. 2, the two target bins are placed side-by-side on the target line as shown in Figs. 12(c) and 13(c). Regarding the coherency of motion, for the case of modulated interaction, it can be seen that the trajectories are more directed toward the targets, and the individual with CP covers a wide range of workspace (full range of needed workspace on the target line which was $17 \mathrm{~cm}$ at the task-side robot) to laterally separate and distribute the objects; in addition, the deviation from the target line in the $X$-direction ("overshoot") is minimal, which indicates that the individual with CP was capable of stopping accurately at the target locations to perform the task. However, for the case of normal interaction, several nonsmooth deviations can be observed. Based on the histograms shown in Fig. 14, for the modulated interaction, the CP individual had the control to hover over top of the targets (which resulted in brighter spots on the target line) rather than overshoot them.

In addition, for the case of normal interaction, the individual with $\mathrm{CP}$ was only able to use a small portion of the target line $(42 \%$ of the defined needed full range of motion on the target line) to distribute the objects along the line. Also, due to the inability of the individual with CP to stop accurately over the target locations nonsmooth movements on the target line and overshoots can be observed in the motion trajectory. The histogram of the normal interaction is taller in the $X$-direction

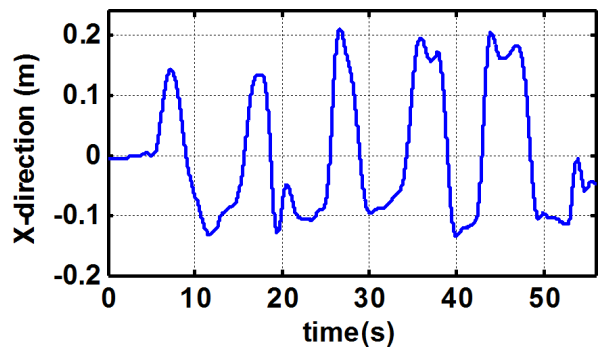

(a)

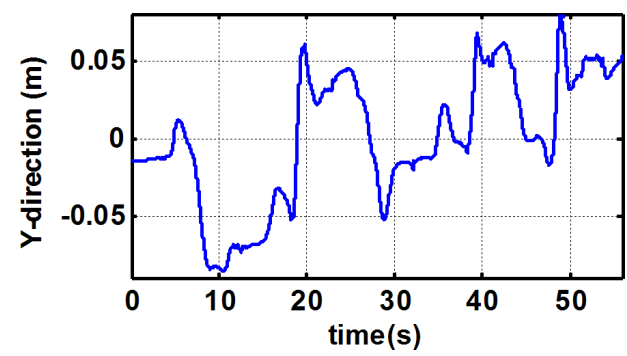

(b)

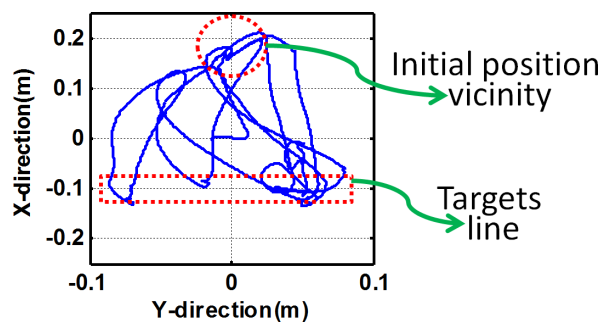

(c)

Fig. 12. Motion trajectories for the modulated interaction: (a) $X$-direction, (b) $Y$-direction and (c) 2D movement.

(because of the overshoots) and is thinner in the $Y$-direction (due to the limited capability in covering the needed workspace especially on the target line). The improved motion coherency corresponds with better coordination and better motion capabilities.

In summary, using the proposed system, the individual with CP was capable of using her convenient workspace to reach and perform the tasks, the motions were smoother in both position and velocity domains and the individual with CP showed better coordination accuracy and motion execution while performing tasks. This was realized by using the proposed telerobotic architecture, which assisted the individual with $\mathrm{CP}$ to manage the involuntary actions and enhance her movement capabilities.

The results illustrate the effectiveness of the proposed system and show that using the telerobotic architecture, it is possible to augment the motion capabilities of an individual with $\mathrm{CP}$ and assist them to provide smoother and more controlled movements for performing tasks. This presents a new assistive compensatory paradigm for people living with CP. 


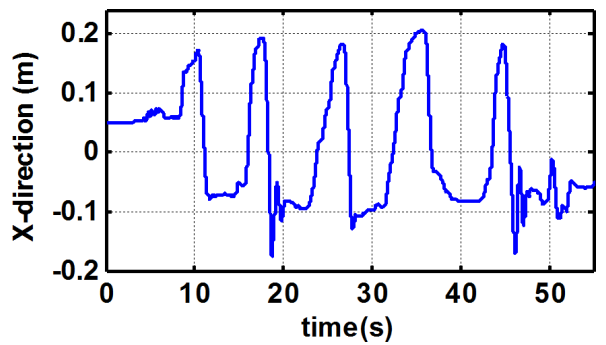

(a)

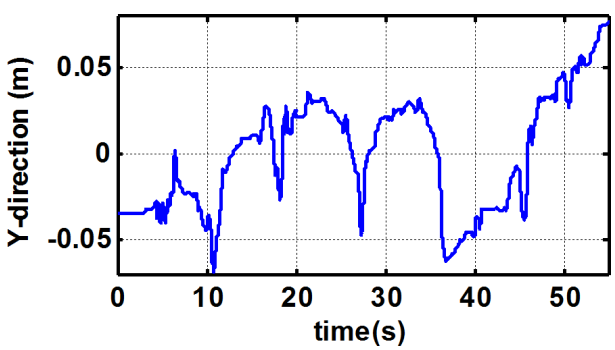

(b)

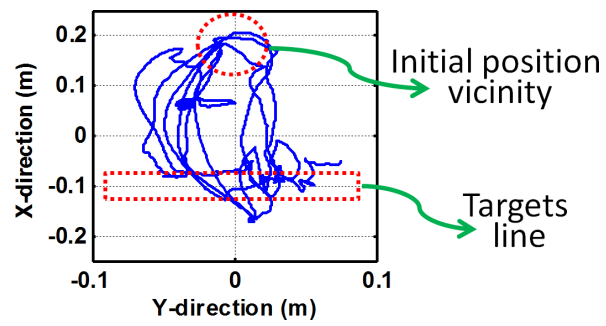

(c)

Fig. 13. Motion trajectories for the normal interaction (when the proposed actions are disabled): (a) $X$-direction, (b) $Y$-direction and (c) 2D movement.

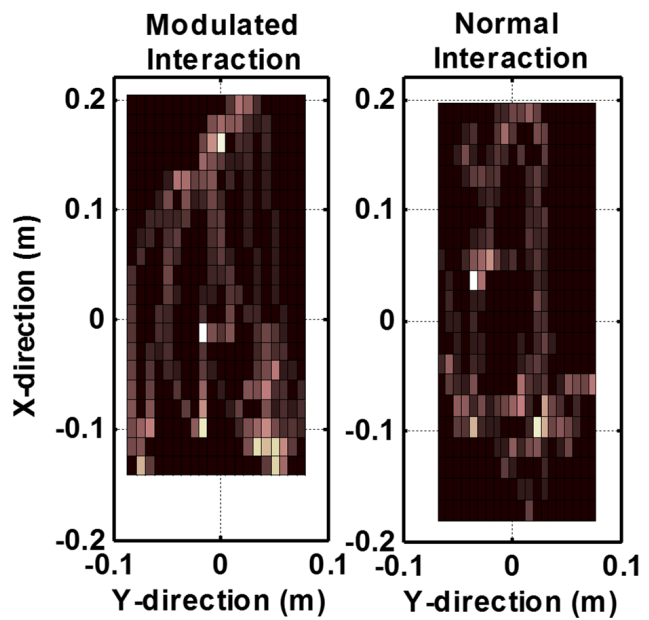

Fig. 14. The histogram of movement for (left) modulated and (right) normal interactions. The histograms show the amount of time spent on different locations of workspace; the brighter a pixel is, the higher amplitude of the histogram, and the longer length of time spent at that spot.

\section{Conclusion}

In this paper, a new telerobotic architecture is proposed that enhances physical interaction with real objects for individuals living with CP. The design of the proposed architecture is specific for the neurological motor deficit causing coordination issues and involuntary motions. The system is composed of four main components, namely: the user-side robot, the task-side robot, the virtual assistive algorithm, and the task environment. The proposed system has a triple-action design. It extracts the voluntary movements of the user to be transferred from the user-side robot to the task-side robot, then it corrects the motion range to fit the transferred motion within the needed workspace of the task, and finally it provides the user with a resistive force field implemented in high-frequency motion domain to only dissipate involuntary energy of the hand motion. The proposed telerobotic architecture is motivated by evidence showing that interaction with physical environments is crucial for individuals with CP to help prevent various secondary symptoms and further sensorimotor deficits. The proposed architecture is implemented using a Quanser upper-limb rehabilitation robot and a Phantom Premium robot. The implemented system was initially evaluated for a nondisabled participant where various functions of the designed architecture were evaluated. Afterwards, the system was studied for an individual who lives with CP to evaluate potential benefits that can be achieved from the point of view of interaction enhancement. The results support the capability of the system in enhancing physical interaction for the individual with CP through the designed tripleaction architecture. This work suggests that the designed system could be used as a new compensatory assistive platform for people living with CP. Future tests with individuals with $\mathrm{CP}$ will help to evaluate the long-term effect of the proposed system and define a range for the system coefficients.

\section{Acknowledgments}

This research was supported by the Natural Sciences and Engineering Research Council (NSERC) of Canada under a Collaborative Research and Development Grant \# CRDPJ 411603-10, Canadian Institutes of Health Research (CIHR) and NSERC Collaborative Health Research Projects (CHRP) Grant \#314552, NSERC grants RGPIN 372042, RGPIN 03907 and RGPIN 1345, the AGE-WELL Network of Centres of Excellence, CHRP Grant \#316170, the Canada Foundation for Innovation under grant LOF 28241, the Alberta Innovation and Advanced Education Ministry under Small Equipment Grant RCP-12-021, and by in-kind contributions from Quanser Inc. M. Shahbazi and N. Jafari have contributed equally to this work. 


\section{References}

1. C. L. Richards and F. Malouin, Cerebral palsy: Definition, assessment and rehabilitation, Handbook Clinical Neurol. 111 (2012) 183-195.

2. P. Baxter, C. Morris, P. Rosenbaum, N. Paneth, A. Leviton, M. Goldstein, M. Bax, A. Colver, D. Damiano, H. Graham et al., The definition and classification of cerebral palsy, Dev. Med. Child Neurol. 49(s109) (2007) 1-44.

3. C. Morris, Definition and classification of cerebral palsy: A historical perspective, Dev. Med. Child Neurol. 49(s109) (2007) 3-7.

4. A. L. Albright, Spastic cerebral palsy, CNS Drugs 4(1) (1995) 17-27.

5. N. Berker and S. Yalçın, The Help Guide to Cerebral Palsy (2010).

6. J. B. Granild-Jensen, G. Rackauskaite, E. M. Flachs and P. Uldall, Predictors for early diagnosis of cerebral palsy from national registry data, Dev. Med. Child Neurol. (2015).

7. L. A. Koman, B. P. Smith and J. S. Shilt, Cerebral palsy, Lancet 363 (9421) (2004) 1619-1631.

8. J. Vauclair, Phylogenetic approach to object manipulation in human and ape infants, Human Dev. 27(5-6) (1984) 321-328.

9. J. R. Flanagan, M. C. Bowman and R. S. Johansson, Control strategies in object manipulation tasks, Curr. Opin. Neurobiol. 16(6) (2006) 650-659.

10. E. Thelen, Motor development as foundation and future of developmental psychology, Int. J. Behav. Dev. 24(4) (2000) 385-397.

11. M. Wright, L. Hunt and O. Stanley, Quantification of object manipulation in children with cerebral palsy, Dev. Neurorehabil. 4(4) (2001) 187-195.

12. R. J. van den Heuvel, M. A. Lexis, G. J. Gelderblom, R. M. Jansens and L. P. de Witte, Robots and ICT to support play in children with severe physical disabilities: A systematic review, Disabil. Rehabil. Assist. Technol. (2015) 1-14.

13. H. A. Ruff, C. McCarton, D. Kurtzberg and H. G. Vaughan Jr, Preterm infants' manipulative exploration of objects, Child Dev. (1984) 1166-1173.

14. L. Alvarez, A. Rios, K. Adams, P. Encarnação and A. Cook, From infancy to early childhood: The role of augmentative manipulation robotic tools in cognitive and social development for children with motor disabilities, in Converging Clinical and Engineering Research on Neurorehabilitation (Springer, 2013), pp. 905-909.

15. A. M. Cook, K. Adams, P. Encarnação and L. Alvarez, The role of assisted manipulation in cognitive development, Dev. Neurorehabil. 15(2) (2012) 136-148.

16. U. M. Ferm, B. K. Claesson, C. Ottesjö and S. Ericsson, Participation and enjoyment in play with a robot between children with cerebral palsy who use aac and their peers, Augment. Altern. Commun. (2015).

17. P. Ljunglöf, S. Larsson, K. Mühlenbock and G. Thunberg, Trik: A talking and drawing robot for children with communication disabilities, DiaHolmia (2009), p. 121.

18. A. M. Cook and K. Adams, The importance of play: At for children with disabilities, in Design and Use of Assistive Technology (Springer, 2010), pp. 33-39.

19. P. Encarnação, L. Alvarez, A. Rios, C. Maya, K. Adams and A. Cook, Using virtual robot-mediated play activities to assess cognitive skills, Disabil. Rehabil.: Assist. Technol. 9(3) (2014) 231-241.

20. A. M. Cook, K. Adams, J. Volden, N. Harbottle and C. Harbottle, Using lego robots to estimate cognitive ability in children who have severe physical disabilities, Disabil. Rehabil.: Assist. Technol. 6(4) (2011) 338-346.

21. A. Cook, P. Encarnação and K. Adams, Robots: Assistive technologies for play, learning and cognitive development, Technol. Disabil. 22(3) (2010) 127-145.

22. S. Roley, J. V. DeLany, C. Barrows, D. Honaker, D. Sava, V. Talley et al., Occupational Therapy Practice Framework: Domain and Process (2008).
23. B. Siciliano and O. Khatib, Springer Handbook of Robotics, Springer Science \& Business Media (Springer, 2008).

24. B. B. Graham, Using an accelerometer sensor to measure human hand motion, $\mathrm{PhD}$ thesis, Massachusetts Institute of Technology (2000).

25. A. O. Andrade, A. A. Pereira, A. P. S. Paixão, G. L. Cavalheiro, M. F. S. de Almeida, S. B. Fenelon and V. C. Dionisio, Human Tremor: Origins, Detection and Quantification (INTECH Open Access Publisher, 2013).

26. E. C. Lu, R. Wang, R. Huq, D. Gardner, P. Karam, K. Zabjek, D. Hebert, J. Boger and A. Mihailidis, Development of a robotic device for upper limb stroke rehabilitation: A user-centered design approach, Paladyn, J. Behavi. Robot. 2(4) (2011) 176-184.

27. E. C. Lu, Development of an upper limb robotic device for stroke rehabilitation, PhD thesis, University of Toronto (2011).

28. http://www.geomagic.com/en/products/phantom-premium/ overview.

29. K. C. Veluvolu, U.-X. Tan, W. T. Latt, C. Shee and W. T. Ang, Bandlimited multiple fourier linear combiner for real-time tremor compensation, in 29th Annual Int. Conf. on Engineering in Medicine and Biology Society. (EMBS 2007) (IEEE, 2007), pp. 28472850.

30. K. Veluvolu and W. Ang, Estimation and filtering of physiological tremor for real-time compensation in surgical robotics applications, Int. J. Med. Robot. Comput. Assist. Surg. 6(3) (2010) 334-342.

31. K. Veluvolu, W. Latt and W. Ang, Double adaptive bandlimited multiple fourier linear combiner for real-time estimation/filtering of physiological tremor, Biomed. Signal Process. Control 5(1) (2010) 37-44.

32. S. F. Atashzar, A. Saxena, M. Shahbazi and R. V. Patel, Involuntary movement during haptics-enabled robotic rehabilitation: Analysis and control design, in IEEE/RSJ Int. Conf. on Intelligent Robots and Systems (IROS 2014) (IEEE, 2014), pp. 3561-3566.

33. S. Wang, Y. Gao, J. Zhao and H. Cai, Adaptive sliding bandlimited multiple fourier linear combiner for estimation of pathological tremor, Biomed. Signal Process. Control 10 (2014) 260-274.

34. C. N. Riviere, R. S. Rader and N. V. Thakor, Adaptive cancelling of physiological tremor for improved precision in microsurgery, IEEE Trans. Biomed. Eng. 45(7) (1998) 839-846.

35. C. N. Riviere, S. G. Reich and N. V. Thakor, Adaptive fourier modeling for quantification of tremor1, J. Neurosci. Methods 74(1) (1997) 77-87.

36. S. Tatinati, K. C. Veluvolu and W. T. Ang, Multistep prediction of physiological tremor based on machine learning for robotics assisted microsurgery, IEEE Trans. Cybernet. 45(2) (2015) 328-339.

37. K. C. Veluvolu, S. Tatinati, S.-M. Hong and W. T. Ang, Multistep prediction of physiological tremor for surgical robotics applications, IEEE Trans. Biomed. Eng. 60(11) (2013) 3074-3082.

38. K. C. Veluvolu and W. T. Ang, Estimation of physiological tremor from accelerometers for real-time applications, Sensors 11(3) (2011) 3020-3036.

39. R. Huq, R. Wang, E.-H. Lu, D. Hebert, H. Lacheray and A. Mihailidis, Development of a fuzzy logic based intelligent system for autonomous guidance of post-stroke rehabilitation exercise, in IEEE Int. Conf. on, Rehabilitation Robotics (ICORR) (IEEE, 2013), pp. 1-8.

40. A. C. Rodríguez, C. Roda, F. Montero, P. González and E. Navarro, An interactive fuzzy inference system for teletherapy of older people, Cognit. Comput. 1-18.

41. R. Palisano, P. Rosenbaum, D. Bartlett and M. Livingston, Gross motor function classification system expanded and revised (gmfcs-e \& r), CanChild Center for Childhood Disability Research, McMaster University (2007).

42. A.-C. Eliasson, L. Krumlinde-Sundholm, B. Rösblad, E. Beckung, M. Arner, A.-M. Öhrvall and P. Rosenbaum, The manual ability classification system (macs) for children with cerebral palsy: Scale development and evidence of validity and reliability, Dev. Med. Child Neurol. 48(7) (2006) 549-554. 


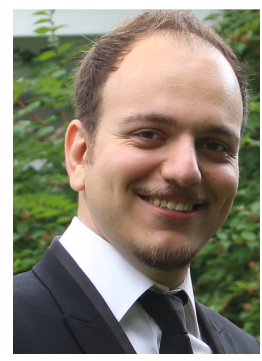

S. Farokh Atashzar obtained his B.Sc. degree in Electrical Engineering/Control Systems from K. N. Toosi University of Technology, Tehran, Iran, in 2008 and his M.Sc. degree in Mechatronics from Amirkabir University of Technology, Tehran, Iran, in 2011. Farokh joined Western University, London, Ontario, Canada, in 2011 to pursue his Ph.D. degree under the supervision of Dr. Rajni Patel. In 2011, he was a doctoral trainee in the NSERC CREATE program in Computer-Assisted Medical Interventions (CAMI). Farokh received his Ph.D. degree in Electrical and Computer Engineering from Western University in 2016. During his Ph.D., Farokh received several awards including the Ontario Graduate Scholarship (OGS) in 2013. He was a visiting research scholar at the University of Alberta, Canada, in 2014. Currently, Farokh is a postdoctoral research associate in the Department of Electrical and Computer Engineering at Western University and Canadian Surgical Technologies and Advanced Robotics (CSTAR), London, Ontario, Canada..

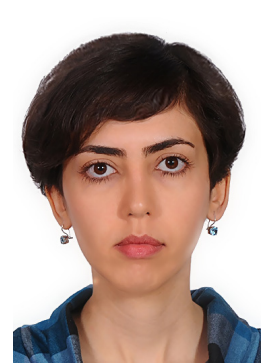

Nooshin Jafari received her M.Sc. degree in Biomedical Engineering from Chalmers University of Technology, Sweden, and the B.Sc. degree in Electronic Engineering from Azad University of Najafabad, Iran. She is a Research Associate in the Assistive Technologies Lab in the Faculty of Rehabilitation Medicine, University of Alberta, Canada. She has been involved in multidisciplinary projects in the areas of robotic assistive technology development and evaluation for children with disabilities, in collaboration with the Control Systems Lab in the Department of Electrical and Computer Engineering and the Alexander Kostov Lab at the Glenrose Rehabilitation Hospital. Her current research interests are assistive technologies and haptic system development, and design of computeraided diagnosis systems.

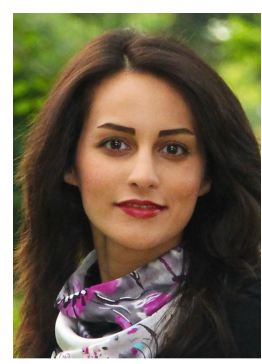

Mahya Shahbazi received her B.Sc. degree in Electrical Engineering from K. N. Toosi University of Technology, Tehran, Iran, in 2008, and the M.Sc. degree in Mechatronics from Amirkabir University of Technology, Tehran, in 2011. She joined the Department of Electrical and Computer Engineering at Western University, London, Ontario, Canada, in 2011 to pursue her Ph.D. degree under the supervision of Dr. Rajni Patel. She was a doctoral trainee in the NSERC CREATE program in Computer-Assisted Medical Interventions in 2012-2013. She was a visiting research scholar at the University of Alberta, Canada. Mahya was among the very few international students at Western University awarded an Ontario Graduate Scholarship in 2014. Mahya completed her Ph.D. degree in Electrical and Computer Engineering in January 2017. She is currently a postdoctoral research associate in the Department of Electrical and Computer Engineering at Western University and Canadian Surgical Technologies and Advanced Robotics (CSTAR), London, Ontario, Canada.

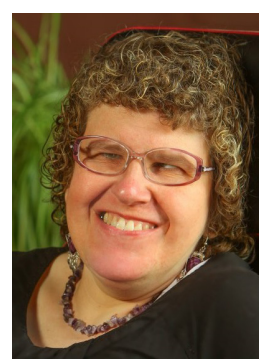

Heidi Janz is an adjunct professor with the John Dossetor Heath Ethics Centre at the University of Alberta, Canada, specializing in the field of Disability Ethics. Her PhD Dissertation was nominated for a Governor General's Award, and she is the winner of the 2010 Tanis Doe Award for Canadian Disability Study and Culture. She was named Global TV's Woman of Vision in May 2013. In her "other life," Heidi Janz is a writer/playwright and filmmaker based in Edmonton Alberta. Her creative work focusses on making the experiences of people with disabilities accessible to audiences made up of both people with disabilities and people who are temporarily able-bodied. To-date, she has written and produced four stage plays. Her first short-film, We Regret to Inform You... (2015), co-written and co-directed with Eva Colmers, and produced by the National Film Board, offers an unsentimental and unapologetic look at what it means to be both "disabled" and "productive" as it follows Heidi through her everyday life. The film was a double Rosie Award winner at the 2016 Alberta Motion Picture Industry Association (AMPIA) Awards, winning Best Direction for a Documentary Short and Best Editing for a Documentary. It also won the EDA Award for Best Female-Directed Documentary Short at the 2016 DOXA Documentary Film Festival. Heidi Janz also has cerebral palsy.

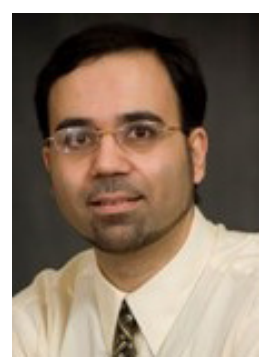

Mahdi Tavakoli is an Associate Professor in the Department of Electrical and Computer Engineering, University of Alberta, Canada. He received his BSc and MSc degrees in Electrical Engineering from Ferdowsi University and K.N. Toosi University, Iran, in 1996 and 1999, respectively. He received his $\mathrm{PhD}$ degree in Electrical and Computer Engineering from the University of Western Ontario, Canada, in 2005. In 2006, he was a post-doctoral researcher at Canadian Surgical Technologies and Advanced Robotics (CSTAR), Canada. In 2007-2008, he was an NSERC PostDoctoral Fellow at Harvard University, USA. Dr. Tavakoli's research interests broadly involve the areas of robotics and systems control. Specifically, his research focuses on haptics and teleoperation control, medical robotics, and image-guided surgery. Dr. Tavakoli is the lead author of Haptics for Teleoperated Surgical Robotic Systems (World Scientific, 2008).

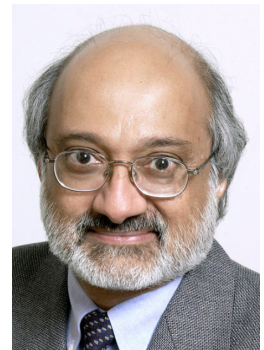

Rajni V. Patel received the $\mathrm{PhD}$ degree in Electrical Engineering from the University of Cambridge, England, in 1973 and currently holds the position of Distinguished University Professor and Tier-1 Canada Research Chair in the Department of Electrical and Computer Engineering with cross appointments in the Department of Surgery and the Department of Clinical Neurological Sciences in the Schulich School of Medicine and Dentistry at Western University, London, Ontario, Canada. Dr. Patel also serves as Director of Engineering for Canadian Surgical Technologies \& Advanced Robotics (CSTAR). He is a Life Fellow of IEEE, and a Fellow of ASME, the Royal Society of Canada and the Canadian Academy of Engineering. He has served on the editorial boards of the IEEE Transactions on Robotics, the IEEE/ASME Transactions on Mechatronics, the IEEE Transactions on Automatic Control, and Automatica, and is currently on the editorial boards of the International Journal of Medical Robotics and Computer Assisted Surgery and the Journal of Medical Robotics Research. 


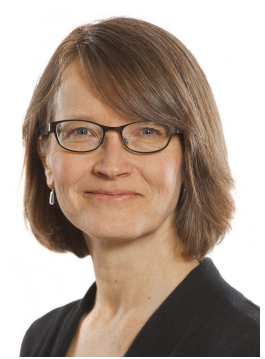

Kim Adams holds a joint position as Assistant Professor of Rehabilitation Medicine at the University of Alberta, and Clinician Scientist at the Glenrose Rehabilitation Hospital, in Edmonton, Canada. She received her PhD degree in Rehabilitation Science from the University of Alberta, Canada, in 2011. She is a pastRESNA (Rehabilitation Engineering and Assistive Technology Society of North America) board member and past-chair of RESNA's special interest group on internationally appropriate technology. She has 20 years of clinical experience in the area of assistive technology. Dr. Adams's research interests are assistive technology design, development and evaluation, use of assistive robots for children with physical disabilities to engage in play and learning activities, and augmentative and alternative communication. 